\title{
Existence of Solutions for Fractional Boundary Value Problem Involving p-Laplacian Operator
}

Ghader Mohmmed Alqurishi

Jeddah, KSA

Email: ghadeerm2172@gmail.com

How to cite this paper: Alqurishi, G.M. (2021) Existence of Solutions for Fractional Boundary Value Problem Involving pLaplacian Operator. Open Access Library Journal, 8: e7192.

https://doi.org/10.4236/oalib.1107192

Received: January 27, 2021

Accepted: February 21, 2021

Published: February 24, 2021

Copyright $\odot 2021$ by author(s) and Open Access Library Inc.

This work is licensed under the Creative Commons Attribution International License (CC BY 4.0).

http://creativecommons.org/licenses/by/4.0/

\begin{abstract}
In this paper, we investigate the question of existence of nonnegative solution for some fractional boundary value problem involving $\mathrm{p}$-Laplacian operator, The results presented in this thesis are based on fixed point theorem, more precisely, Krasnosilski fixed point theorem, on the cones to prove the existence of a fixed point for a mathematics operator and that fixed point is a solution to the given fractional equation by combining some properties of the associated Green function. We will study the problem of boundary value and find the unique solution. We will present definition and properties of Riemann-Liouville derivatives, the Guo-Krasnoselskii fixed point theorem, the Green Function to find the positive solution of problem involving p-Laplacian. The main idea is to transfer the given problem to some integral equation; from this integral equation we define an operator with Green function. We prove that this operator is completely continuous and uniformly bounded which yields by Arzela Ascoli Theorem that this operator is compact. So by Krasnosilski fixed point theorem can find the result. This type of problem is very interesting in different areas such as biophysics, astronomy and others. Note that this problem can be extended to the fractional Hadamard derivative and to the Leggett Williams fixed point theorem; moreover, it can be the content of a rigorous thesis topic.
\end{abstract}

\section{Subject Areas}

Ordinary Differential Equation, Partial Differential Equation

\section{Keywords}

Fractional Calculus, Riemann-Liouville Fractional, p-Laplacian Operator, Krasnoselskii Fixed-Point Theorem 


\section{Introduction}

Fractional order differential and integral operators extensively appear in the mathematical modeling of various scientific and engineering phenomena. The main advantage for using these operators is their nonlocal nature, which can describe the past history of processes and materials involved in the phenomena. Mathematical theory of fractional derivative has in the past few decades rapidly developed into an important and separate field of applied mathematics. Important area of applications of this theory lies in viscoelastic materials, as well as in many fields of science and engineering including fluid flow, rheology, diffusive transport, electromagnetic theory and probability. This type of derivatives appears in the study of differential equations which is a wide field in pure and applied mathematics, physics, meteorology, and engineering. All of these disciplines are concerned with the properties of differential equations of various types. Fractional differential equations equipped with classical, nonlocal and integral boundary conditions have recently been investigated by many researchers. One can witness overwhelming interest in the study of nonlocal nonlinear fractional-order boundary value problems (FBVPs) in the related literature. Boundary value problems of fractional differential equations involving Riemann-Liouville, Caputo, Hadamard type derivatives and different kinds of boundary conditions have been extensively studied by many researchers. The area of study for such problems includes existence and uniqueness of solutions, stability and oscillatory properties, analytic and numerical methods. The main idea in our study, is to transfer the given differential equation to an integral equation, to investigate the question of existence of nonnegative solution for some fractional boundary value problems involving p-Laplacian operator. Our approach is based on fixed point theorem; more precisely, we use the well known Krasnosilski fixed point theorem to prove our main result. The tools of our study include some standard fixed point theorems such as Banach's contraction mapping principle, Leray-Schauder nonlinear alternative, Krasnoselskii's fixed point theorem.

Let $\alpha, \beta \in(1,2], a, b \in(0,1)$ and $p>1$, the purpose of this chapter is to study the following fractional boundary value problem

$$
\begin{array}{ll}
D^{\beta}\left(\varphi_{p}\left(D^{\alpha} u(t)\right)\right)=h(t) f(u(t)), & 0<t<1, \\
u(0)=D^{\alpha} u(0)=0, u(1)=\lambda u(a), & D^{\alpha} u(1)=\mu D^{\alpha} u(b),
\end{array}
$$

where $\mu \geq 0,0<a, b<1, \quad \varphi_{p}(x)=|x|^{p} x, D^{\alpha} \quad$ (resp. $\left.D^{\beta}\right)$ is the standard Riemann-Liouville fractional differential operator of order $\alpha$ (resp. of order $\beta$ ). $h$ is a measurable function on $[0,1]$, and the nonlinear term $f$ is a continuous function.

The theory of fractional calculus may be used in the description of memory and hereditary properties of various materials and processes. The mathematical modelling of systems and processes in the fields of physics, chemistry, aerodynamics, electrodynamics of complex medium, polymer rheology, Consequently, the subject of fractional differential equations is gaining more importance and 
attention. There has been significant development in ordinary and partial differential equations involving Riemann Liouville fractional derivatives. For details and examples, one can see the monographs [1] [2] and references therein. Bai [3] considered the following three-point boundary value problem of the fractional order differential equation

$$
\begin{aligned}
& D^{\alpha} u(t)+f(t, u(t))=0,0<t<1, \\
& u(0)=0, \quad u(1)=\beta u(\mu),
\end{aligned}
$$

where $1<\alpha \leq 2,0<\mu<1$ is such that $0<\beta \mu^{\alpha-1}<1$. By using the contraction map principle and fixed-point index theory, the author investigated the existence and uniqueness of positive solutions for problem (2). Xu, Jiang et al. [4] deduced some new properties of Green's function of (2). By using some fixed point theorems, they obtained the existence, uniqueness and multiplicity of positive solutions to singular problems. Recently, boundary value problems of nonlinear fractional differential equations have aroused considerable attention. Many authors pay attention to the existence results for boundary value problems of nonlinear fractional differential equations by means of some fixed point theorems, such as the Krasnoselskii fixed-point theorem (see [1] [5]), the Leggett-Williams fixed-point theorem (see [6]), and the Schauder fixed-point theorem (see [7]). To the best of our knowledge, there are few papers devoted to investigating nonlinear fractional differential equations involving p-Laplacian operator (see [8]). In the rest of this paper, we write problem (1) as an equivalent integral equation and then, by using some properties of the associated Green function and the Guo-Krasnoselskii fixed point theorem, we obtain our main result asserting existence of nontrivial nonnegative solutions to problem (1). Finally, an example is given to demonstrate the effectiveness of the obtained result.

\section{Preliminaries and Basic Definitions.}

We present in this section some necessary definitions and lemmas that we will use in the rest of this paper.

Definition 2.1. Let $n$ be a positive integer and $n-1<\sigma \leq n$.

1) The Riemann-Liouville's derivatives of order $\alpha$ for a function $f$ is denoted by $D_{x}^{\alpha} f(x)$, and is defined by:

$$
D_{x}^{\alpha} f(x)=\frac{1}{\Gamma(n-\alpha)}\left(\frac{\mathrm{d}}{\mathrm{d} x}\right)^{n} \int_{0}^{x} \frac{f(\xi)}{(x-\xi)^{1-n+\alpha}} \mathrm{d} \xi, x>0,
$$

2) The Riemann-Liouville integral of order $\sigma$ for a function $f$ is defined by:

$$
I_{x}^{\alpha} f(x)=\frac{1}{\Gamma(\alpha)} \int_{0}^{x} \frac{f(\xi)}{(x-\xi)^{1-\alpha}} \mathrm{d} \xi, x>0,
$$

provided that the right-hand side is pointwise defined on $(0, \infty)$.

Lemma 2.2. Let $n$ be a positive integer and $n-1<\alpha \leq n$, then

$$
I_{0^{+}}^{\alpha} D_{0^{+}}^{\alpha} u(t)=u(t)+c_{0}+c_{1} t+c_{2} t^{2}+\cdots+c_{n-1} t^{n-1}
$$


for some $c_{i} \in R, i=0,1,2,3, \cdots, n-1$.

Lemma 2.3. (see [3]). Let $h \in C[0,1]$, and assume that $1-\lambda^{\alpha-1}>0$, then the following problem

$$
\left\{\begin{array}{l}
D^{\alpha} u(t)+h(t)=0,0<t<1, \\
u(0)=0, u(1)=\lambda u(a)
\end{array}\right.
$$

has a unique solution which is given by

$$
u(t)=\int_{0}^{1} K(t, s) h(s) \mathrm{d} s,
$$

where

$$
K(t, s)= \begin{cases}\frac{t^{\alpha-1}(1-s)^{\alpha-1}-\lambda t^{\alpha-1}(a-s)^{\alpha-1}}{\Gamma(\alpha)\left(1-\lambda a^{\alpha-1}\right)}-\frac{(t-s)^{\alpha-1}}{\Gamma(\alpha)}, & \text { if } 0 \leq s \leq \min (t, a) \leq 1, \\ \frac{t^{\alpha-1}(1-s)^{\alpha-1}-\left(1-\lambda a^{\alpha-1}\right)(t-s)^{\alpha-1}}{\Gamma(\alpha)\left(1-\lambda a^{\alpha-1}\right)}, & \text { if } 0 \leq a \leq s \leq t \leq 1, \\ \frac{t^{\alpha-1}(1-s)^{\alpha-1}-\lambda t^{\alpha-1}(a-s)^{\alpha-1}}{\Gamma(\alpha)\left(1-\lambda a^{\alpha-1}\right)}, & \text { if } 0 \leq t \leq s \leq a \leq 1, \\ \frac{t^{\alpha-1}(1-s)^{\alpha-1}}{\Gamma(\alpha)\left(1-\lambda a^{\alpha-1}\right)}, & \text { if } 0 \leq \max (t, a) \leq s \leq 1 .\end{cases}
$$

Lemma 2.4. Let $h \in C[0,1]$, and assume that $1-\mu^{p-1} b^{\beta-1}>0$, then the following problem

$$
\left\{\begin{array}{l}
D^{\beta}\left(\varphi_{p}\left(D^{\alpha} u(t)\right)\right)=h(t), 0<t<1, \\
u(0)=D^{\alpha} u(0)=0, u(1)=\lambda u(a), D^{\alpha} u(1)=\mu D^{\alpha} u(b),
\end{array}\right.
$$

has a unique solution which is given by:

$$
u(t)=\int_{0}^{1} K(t, s) \varphi_{q}\left(\int_{0}^{1} G(s, v) h(v) \mathrm{d} v\right) \mathrm{d} s
$$

where

$$
G(t, s)= \begin{cases}\frac{t^{\beta-1}(1-s)^{\beta-1}-\mu^{p-1} t^{\beta-1}(b-s)^{\beta-1}}{\Gamma(\beta)\left(1-\mu^{p-1} b^{\beta-1}\right)}-\frac{(t-s)^{\beta-1}}{\Gamma(\beta)}, & \text { if } 0 \leq s \leq \min (t, b) \leq 1, \\ \frac{t^{\beta-1}(1-s)^{\beta-1}-\left(1-\mu^{p-1} b^{\beta-1}\right)(t-s)^{\beta-1}}{\Gamma(\beta)\left(1-\mu^{p-1} b^{\beta-1}\right)}, & \text { if } 0 \leq b \leq s \leq t \leq 1, \\ \frac{t^{\beta-1}(1-s)^{\beta-1}-\mu^{p-1} t^{\beta-1}(b-s)^{\beta-1}}{\Gamma(\beta)\left(1-\mu^{p-1} b^{\beta-1}\right)}, & \text { if } 0 \leq t \leq s \leq b \leq 1, \\ \frac{t^{\beta-1}(1-s)^{\beta-1}}{\Gamma(\beta)\left(1-\mu^{p-1} b^{\beta-1}\right)}, & \text { if } 0 \leq \max (t, b) \leq s \leq 1 .\end{cases}
$$

Proof. Let $t \in(0,1)$, and assume that $D^{\beta}\left(\varphi_{p}\left(D^{\alpha} u(t)\right)\right)=h(t)$. Then from Lemma 2.2 we have

$$
\varphi_{p}\left(D^{\alpha} u(t)\right)=I^{\beta} h(t)+c_{1} t^{\beta-1}+c_{2} t^{\beta-2}, c_{1}, c_{2} \in \mathbb{R} .
$$


$D^{\alpha} u(0)=0$, implies that $c_{2}=0$.

From the boundary condition $D^{\alpha} u(1)=\mu D^{\alpha} u(b)$, we obtain

$$
c_{1}=-\int_{0}^{1} \frac{(1-s)^{\beta-1}}{\Gamma(\beta)\left(1-\mu^{p-1} b^{\beta-1}\right)} h(s) \mathrm{d} s+\int_{0}^{b} \frac{\mu^{p-1}(b-s)^{\beta-1}}{\Gamma(\beta)\left(1-\mu^{p-1} b^{\beta-1}\right)} h(s) \mathrm{d} s
$$

Put $c_{1}$ and $c_{2}$ in Equation (6) we find

$$
\begin{aligned}
\varphi_{p}\left(D^{\alpha} u(t)\right)= & \int_{0}^{t} \frac{(t-s)^{\beta-1}}{\Gamma(\beta)} h(s) \mathrm{d} s-\int_{0}^{1} \frac{t^{\beta-1}(1-s)^{\beta-1}}{\Gamma(\beta)\left(1-\mu^{p-1} b^{\beta-1}\right)} h(s) \mathrm{d} s \\
& +\int_{0}^{b} \frac{\mu^{p-1} t^{\beta-1}(b-s)^{\beta-1}}{\Gamma(\beta)\left(1-\mu^{p-1} b^{\beta-1}\right)} h(s) \mathrm{d} s \\
= & -\int_{0}^{1} G(t, s) h(s) \mathrm{d} s,
\end{aligned}
$$

where $G(t, s)$ is given by Equation (5). So, boundary value problem (4) is equivalent to

$$
\begin{aligned}
& D^{\alpha} u(t)+\varphi_{q}\left(\int_{0}^{1} G(t, s) h(s) \mathrm{d} s\right)=0,0<t<1, \\
& u(0)=0, \quad u(1)=\lambda u(a) .
\end{aligned}
$$

Consequently, From Lemma 2.3, the unique solution of problem (4) is given by

$$
u(t)=\int_{0}^{1} K(t, s) \varphi_{q}\left(\int_{0}^{1} G(t, s) h(s) \mathrm{d} s\right) \mathrm{d} s .
$$

The proof is completed.

In the next lemmas, we present important properties of the Green functions $G$ and $K$.

Lemma 2.5. (See [6]) The functions $G$ and $K$ defined respectively by equations (3) and (5) are nonnegative continuous on $[0,1] \times[0,1]$, moreover, for all $t, s \in[0,1]$, we have

$$
G(t, s) \leq G(s, s),
$$

and

$$
K(t, s) \leq K(s, s) .
$$

Lemma 2.6. There exist positive continuous functions $\sigma_{1}$ and $\sigma_{2}$ such that for all $s \in[0,1]$, we have

$$
\min _{a \leq t \leq 1} K(t, s) \geq \sigma_{1}(s) K(s, s),
$$

and

$$
\min _{b \leq t \leq 1} G(t, s) \geq \sigma_{2}(s) G(s, s) .
$$

Proof. To simplify, we denote

$$
K(t, s)= \begin{cases}k_{1}(t, s), & \text { if } 0 \leq s \leq \min (t, a) \leq 1, \\ k_{2}(t, s), & \text { if } 0 \leq a \leq s \leq t \leq 1, \\ k_{3}(t, s), & \text { if } 0 \leq t \leq s \leq a \leq 1, \\ k_{4}(t, s), & \text { if } 0 \leq \max (t, a) \leq s \leq 1 .\end{cases}
$$


So, if $a \leq t \leq 1$, then

$$
\begin{gathered}
K(t, s)= \begin{cases}k_{1}(t, s), & \text { if } 0 \leq s \leq a \leq t \leq 1, \\
k_{2}(t, s), & \text { if } 0 \leq a \leq s \leq t \leq 1, \\
k_{4}(t, s), & \text { if } 0 \leq a \leq t \leq s \leq 1 .\end{cases} \\
\min _{a \leq t \leq 1} K(t, s)= \begin{cases}\min _{a \leq t \leq 1}\left(k_{1}(t, s)\right), & \text { if } 0 \leq s \leq a, \\
\min _{a \leq t \leq 1}\left(k_{2}(t, s), k_{4}(t, s)\right), & \text { if } a \leq s \leq 1,\end{cases} \\
\begin{cases}k_{1}(1, s), & \text { if } 0 \leq s \leq a, \\
\min _{a \leq t \leq 1}\left(k_{2}(1, s), k_{4}(a, s)\right), & \text { if } a \leq s \leq 1 .\end{cases}
\end{gathered}
$$

Put

$$
\sigma_{1}(s)= \begin{cases}\frac{k_{1}(1, s)}{K(s, s)}, & \text { if } 0 \leq s \leq a, \\ \frac{\min _{a \leq t \leq 1}\left(k_{2}(1, s), k_{4}(a, s)\right)}{K(s, s)}, & \text { if } a \leq s \leq 1,\end{cases}
$$

Then, it is easy to see that for all $s \in[0,1]$, we have

$$
\min _{a \leq t \leq 1} K(t, s) \geq \sigma_{1}(s) K(s, s) .
$$

Inequality (11) can be proved similarly.

The following lemma is fundamental in the proofs of our main results.

Lemma 2.7. (Guo-Krasnoselskii) Let $(E,\|\|$.$) be a Banach space, and P \subset E$ be a cone. Assume $\Omega_{1}, \Omega_{2}$ are bounded open subsets of $E$ with $0 \in \Omega_{1}, \Omega_{1} \subset \Omega_{2}$, and let

$$
T: P \cap\left(\overline{\Omega_{2}} \backslash \Omega_{1}\right) \rightarrow P,
$$

be a completely continuous operator such that either

1) $\|T u\| \leq\|u\|$ for $u \in P \bigcap \partial \Omega_{1}$ and $\|T u\| \geq\|u\|$ for $u \in P \bigcap \partial \Omega_{2}$; or

2) $\|T u\| \geq\|u\|$ for $u \in P \bigcap \partial \Omega_{1}$ and $\|T u\| \leq\|u\|$ for $u \in P \bigcap \partial \Omega_{2}$.

Then $T$ has a fixed point in $P \cap\left(\overline{\Omega_{2}} \backslash \Omega_{1}\right)$.

\section{Main Result and Proof}

Throughout this section, we put $\rho=\max (a, b)$, and assume the following hypothesis:

$\left(\mathrm{H}_{1}\right)$ The function $h:[0,1] \rightarrow[0, \infty)$, is a nontrivial Lebesgue integrable function.

$\left(\mathrm{H}_{2}\right)$ The function $f$ is nonnegative continuous such that there exist $r_{2}>r_{1}>0$, satisfying
1) $f(x) \geq \theta_{1} \varphi_{p}\left(r_{1}\right)$, for all $x \in\left[0, r_{1}\right]$.
2) $f(x) \leq \theta_{2} \varphi_{p}\left(r_{2}\right)$, for all $x \in\left[0, r_{2}\right]$,

where 


$$
\theta_{1}=\left(\int_{\rho}^{1} \sigma_{1}(s) K(s, s) \mathrm{d} s\right)^{1-p}\left(\int_{\rho}^{1} \sigma_{2}(\tau) G(\tau, \tau) h(\tau) \mathrm{d} \tau\right)^{-1}
$$

and

$$
\theta_{2}=\left(\int_{0}^{1} K(s, s) \mathrm{d} s\right)^{1-p}\left(\int_{0}^{1} G(\tau, \tau) h(\tau) \mathrm{d} \tau\right)^{-1}
$$

Our main result in this paper is the following theorem.

Theorem 3.1. Assume that hypotheses $\left(\mathrm{H}_{1}\right)-\left(\mathrm{H}_{2}\right)$ are satisfied. If

$$
1-\lambda a^{\alpha-1}>0 \text {, and } 1-\mu^{p-1} b^{\beta-1}>0,
$$

then the fractional boundary value problem (1) has a nontrivial nonnegative solution $u$ such that $r_{1} \leq\|u\| \leq r_{2}$.

Proof. Let $E=C([0,1], \mathbb{R})$ be the Banach space of all continuous real functions on $[0,1]$, which is equipped with the maximum norm

$$
\|u\|=\max _{0 \leq t \leq 1}|u(t)| \text {. }
$$

Then $(E,\|u\|)$ is a real Banach space. Let $P=\{u \in E, u(t) \geq 0, \forall t \in[0,1]\}$, then $P$ is a cone in $E$. In the sequel, for $\sigma>0$, let $B_{\rho}:=\{x \in E:\|x\|<\sigma\}$, and define the operator $T: P \rightarrow E$, by

$$
T u(t)=\int_{0}^{1} K(t, s)\left(\int_{0}^{1} G(t, \tau) h(\tau) f(u(\tau)) \mathrm{d} \tau\right)^{\frac{1}{p-1}} \mathrm{~d} s .
$$

From Lemma 2.4, $u$ is a nonnegative solution for problem (1), if and only if it is a fixed point of the operator $T$. Moreover the functions $f, h, K$ and $G$ are nonnegative, we see that the operator $T$ maps $P$ into itself. On the other hand, by using the Arzela-Ascoli theorem, we can prove that $T: P \rightarrow P$ is completely continuous.

Now, let $\rho=\max (a, b)$, and let $u \in P \bigcap \partial B_{r_{1}}$, then from Lemma 2.6 and hypothesis $\left(\mathrm{H}_{2}\right)$, we have

$$
\begin{aligned}
T u(t) & =\int_{0}^{1} K(t, s)\left(\int_{0}^{1} G(t, \tau) h(\tau) f(u(\tau)) \mathrm{d} \tau\right)^{\frac{1}{p-1}} \mathrm{~d} s \\
& \geq \int_{\rho}^{1} \sigma_{1}(s) K(s, s)\left(\int_{\rho}^{1} \sigma_{2}(\tau) G(\tau, \tau) h(\tau) f(u(\tau)) \mathrm{d} \tau\right)^{\frac{1}{p-1}} \mathrm{~d} s \\
& \geq r_{1} \theta_{1}{ }^{\frac{1}{p-1}} \int_{\rho}^{1} \sigma_{1}(s) K(s, s) \mathrm{d} s\left(\int_{\rho}^{1} \sigma_{2}(\tau) G(\tau, \tau) h(\tau) \mathrm{d} \tau\right)^{\frac{1}{p-1}} \\
& =\|u\|
\end{aligned}
$$

On the other hand, if $u \in B_{r_{2}}$, then from Lemma 2.6 and hypothesis $\left(\mathrm{H}_{1}\right)$, we obtain

$$
\begin{aligned}
T u(t) & =\int_{0}^{1} K(t, s)\left(\int_{0}^{1} G(t, \tau) h(\tau) f(u(\tau)) \mathrm{d} \tau\right)^{\frac{1}{p-1}} \mathrm{~d} s \\
& \leq \int_{0}^{1} K(s, s)\left(\int_{0}^{1} G(\tau, \tau) h(\tau) f(u(\tau)) \mathrm{d} \tau\right)^{\frac{1}{p-1}} \mathrm{~d} s \\
& \leq \int_{0}^{1} K(s, s)\left(\int_{0}^{1} G(\tau, \tau) h(\tau) f(u(\tau)) \mathrm{d} \tau\right)^{\frac{1}{p-1}} \mathrm{~d} s \\
& =\|u\|
\end{aligned}
$$


Therefore, Lemma 2.7 implies that the operator $T$ has a fixed point $u$ in $P \cap\left(\overline{B_{r_{2}}} \backslash B_{r_{1}}\right)$, that satisfies $r_{1} \leq\|u\| \leq r_{2}$. This completes the proof of Theorem 3.1 .

In the following, we give an example to illustrate the validity of the main result.

Example 3.2. In this example, problem (1) is considered in the special case, precisely, we consider the following fractional boundary value problem

$$
\left\{\begin{array}{l}
D^{\frac{3}{2}}\left(\varphi_{\frac{9}{5}}\left(D^{\frac{4}{3}} u(t)\right)\right)=\sqrt{t} \ln (u(t)+15), 0<t<1, \\
u(0)=D^{\frac{4}{3}} u(0)=0, u(1)=u\left(\frac{1}{8}\right), D^{\frac{4}{3}} u(1)=D^{\frac{4}{3}} u\left(\frac{1}{8}\right) .
\end{array}\right.
$$

Firstly, we have

$$
1-\lambda a^{\alpha-1}=1-\left(\frac{1}{8}\right)^{\frac{1}{3}}=\frac{1}{2}>0 \text {, and } 1-\mu^{p-1} b^{\beta-1}=1-\left(\frac{1}{8}\right)^{\frac{1}{2}}>0 .
$$

On the other hand, $h(t)=\sqrt{t}$, which is nontrivial nonnegative Lebesgue integrable function on $[0,1]$. So, hypothesis $\left(\mathrm{H}_{1}\right)$ is satisfied.

It is not difficult to show that

$$
\sigma_{1}(s)= \begin{cases}\frac{\frac{(1-s)^{\frac{1}{3}}}{2}-\left(\frac{1}{8}-s\right)^{\frac{1}{3}}}{s^{\frac{1}{3}}(1-s)^{\frac{1}{3}}-s^{\frac{1}{3}}\left(\frac{1}{8}-s\right)^{\frac{1}{3}}}, & \text { if } 0 \leq s \leq \frac{1}{8}, \\ \frac{1}{2 s^{\frac{1}{3}},} & \text { if } \frac{1}{8} \leq s \leq 1,\end{cases}
$$

and

$$
\sigma_{2}(s)= \begin{cases}\frac{\frac{\sqrt{1-s}}{2 \sqrt{2}}-\sqrt{\frac{1}{8}-s}}{\sqrt{s} \sqrt{1-s}-\sqrt{s} \sqrt{\frac{1}{8}-s},} & \text { if } 0<s \leq \frac{1}{8}, \\ \frac{1}{2 \sqrt{2} \sqrt{s}}, & \text { if } \frac{1}{8} \leq s \leq 1 .\end{cases}
$$

By a simple computation, we obtain

$$
\theta_{1} \approx 5.8961 \text { and } \theta_{2} \approx 0.1411 \text {. }
$$

By choosing $r_{1}=\frac{1}{3}$, and $r_{2}=80$ we get

3) $f(x) \geq \theta_{1} \varphi_{p}\left(r_{1}\right)$, for all $x \in\left[0, r_{1}\right]$,

and

4) $f(x) \leq \theta_{2} \varphi_{p}\left(r_{2}\right)$, for all $x \in\left[0, r_{2}\right]$. 
Hence, hypothesis $\left(\mathrm{H}_{2}\right)$ is satisfied.

Finally, Theorem 3.1 implies that problem (14) admits a nontrivial nonnegative solution.

\section{Conflicts of Interest}

The author declares no conflicts of interest regarding the publication of this paper.

\section{References}

[1] Krasnoselskii, M.A. (1964) Positive Solutions of Operator Equations. Noordhoff, Groningen.

[2] Miller, K. and Ross, B. (1993) An Introduction to the Fractional Calculus and Fractional Differential Equations. Wiley and Sons, New York.

[3] Bai, Z. (2010) On Positive Solutions of a Nonlocal Fractional Boundary Value Problem. Nonlinear Analysis, 72, 916-924. https://doi.org/10.1016/j.na.2009.07.033

[4] Ghanmi, A. and Horrigue, S. (2019) Existence of Positive Solutions for a Coupled System of Nonlinear Fractional Differential Equations. Ukrainian Mathematical Journal, 71, 39. https://doi.org/10.1007/s11253-019-01623-w

[5] Ghanmi, A. and Horrigue, S. (2018) Existence Results for Nonlinear Boundary Value Problems. FILOMAT, 32, 609-618. https://doi.org/10.2298/FIL1802609G

[6] Wang, J., Xiang, H.J. and Liu, Z. (2009) Positive Solutions for Three-Point Boundary Value Problems of Nonlinear Fractional Differential Equations with p-Laplacian. Far East Journal of Applied Mathematics, 37, 33-47.

[7] Ghanmi, A., Kratou, M. and Saoudi, K. (2018) A Multiplicity Results for a Singular Problem Involving a Riemann-Liouville Fractional Derivative. FILOMAT, 32, 653-669. https://doi.org/10.2298/FIL1802653G

[8] Saoudi, K., Agarwal, P., Kumam, P., Ghanmi, A. and Thounthong, P. (2018) The Nehari Manifold for a Boundary Value Problem Involving Riemann-Liouville Fractional Derivative. Advances in Difference Equations, 2018, Article Number: 263. https://doi.org/10.1186/s13662-018-1722-8 